\title{
Multiple sclerosis and genetic polymorphisms in fibrinogen-mediated hemostatic pathways: a case-control study
}

\author{
Gianmarco Abbadessa ${ }^{1}$ (1) Giuseppina Miele ${ }^{1} \cdot$ Andrea Di Pietro $^{1} \cdot$ Maddalena Sparaco $^{1} \cdot$ Raffaele Palladino $^{2,3}$. \\ Ignazio Armetta ${ }^{4}$. Giovanna D'Elia ${ }^{4}$. Francesca Trojsi ${ }^{~}$ - Elisabetta Signoriello ${ }^{1}$. Giacomo Lus ${ }^{1}$. Luigi Lavorgna ${ }^{1}$. \\ Simona Bonavita ${ }^{1}$
}

Received: 11 February 2021 / Accepted: 10 September 2021 / Published online: 24 September 2021

(c) The Author(s) 2021

\begin{abstract}
Introduction Blood coagulation constituents might exert immunomodulatory functions in the CNS and may trigger neuroinflammation and demyelination. We evaluated whether particular single-nucleotide polymorphisms (SNPs), thought to be involved in fibrinogen-mediated hemostatic pathways, are overrepresented in patients with MS compared with controls. Methods The case-control study consisted of 119 MS patients recruited consecutively at our clinic, and 68 healthy controls. Afterwards, we created a cumulative genetic risk score (CGRS) which included the 5 selected hemostatic risk alleles (BetaFibrinogen 455G/A, Glycoprotein IIIa P1A2, Factor V Leiden, Factor V H2R, and Prothrombin 20210G/A). Multivariate ordinal logistic regression and multivariate multinomial logistic regression were applied to evaluate the effect of CGRS on MS susceptibility.

Results The FGB 455 G/A and Factor V H1299R variants might be associated with MS status, in the recessive and dominant model, respectively. A cumulative association of the five SNPs investigated with the disease was observed.

Discussion We found that MS patients carried more pro-hemostatic variants than healthy controls. An increasing number of unfavorable alleles might increase the likelihood of being in the MS group, in the cumulative analysis. Our findings encourage to evaluating these variants in a larger population-based cohort.
\end{abstract}

Keywords Multiple sclerosis $\cdot$ Fibrinogen $\cdot$ Coagulation $\cdot$ Polymorphisms

\section{Introduction}

Multiple sclerosis (MS) is a chronic inflammatory autoimmune disease that affects the central nervous system (CNS) causing demyelination and axonal damage [1]. Several genetic and environmental factors have been linked to the disease and adaptive immunity, and autoimmune

Gianmarco Abbadessa

gianmarcoabbadessa@live.com

1 Department of Advanced Medical and Surgical Sciences, University of Campania “Luigi Vanvitelli”, Naples, Italy

2 Department of Public Health, University Federico II, Naples, Italy

3 Department of Primary Care and Public Health, Imperial College London, London, UK

4 Clinical and Molecular Pathology, Department of Precision Medicine, University of Campania Luigi Vanvitelli, Naples, Italy phenomena seem to play a pivotal role in its pathogenesis [1]. Since its first pathological description, vascular alterations have been described. Above all, blood-brain barrier (BBB) leakage, fibrin deposition in the perivascular space, vessel occlusion, and cerebral hypoperfusion have been better characterized by subsequent studies [2]. Furthermore, increasing evidence has suggested that blood coagulation constituents might exert immunomodulatory functions in the CNS with critical consequences in fostering and amplifying neuroinflammation and the demyelinating process $[3,4]$. The strongest evidence is available for fibrin(-ogen). It leaks out through the damaged BBB at the earliest stage of MS lesion, preceding demyelination and axonal loss, and spatially correlates with areas of demyelination and axonal damage [5]. In experimental allergic encephalitis (EAE), fibrinogen specifically promotes the activation of macrophages/microglia and favors the recruitment of encephalitogenic T cells in the CNS $[6,7]$. Moreover, fibrin-targeting immunotherapy can be 
selective and efficacious in suppressing neuroinflammation and neurodegeneration in EAE [8].

Due to the key role displayed by fibrin(-ogen) in the extensive cross talk between hemostasis and neuroinflammation, our purpose was to determine whether particular single-nucleotide polymorphisms (SNPs), thought to be involved in fibrinogen-mediated hemostatic pathways, are overrepresented in patients with MS compared with controls. Furthermore, in order to identify genetic factors predicting a worse outcome in MS patients, we investigated whether the frequency of the investigated SNPs was associated with clinical and radiological outcomes at diagnosis.

\section{Methods}

\section{Patients}

This unmatched case-control study consists of 119 relapsing-remitting (RR)-MS patients recruited consecutively at our clinic, and 68 healthy controls. Data on patient characteristics at diagnosis, including Expanded Disability Status Scale (EDSS) and radiological features (number of $\mathrm{T} 2$ lesion $\geqq 9$, presence of spinal cord involvement, and presence of contrast-enhancing lesions), were collected. Exclusion criteria for patients were as follows: history of cerebral and/or cardiovascular ischemic events, thrombotic or bleeding disorders, chronic autoimmune diseases. Controls were recruited among hospital employees. The control group consisted of volunteers without a self-reported history of cerebrovascular or cardiovascular disease, thrombotic or bleeding disorders, or autoimmune chronic disease. As genetic polymorphisms vary between races and populations, all the controls had the same ethnic (Caucasian) and geographic background (people originating from Campania, Southern Italy) as patients.

\section{Selection of SNPs and genotyping}

The assay to assess the genetically determined cardiovascular risk was the Cardiovascular Disease (CVD) 14 (Nuclear Laser Medicine, Naples, IT; for more details, see Supplementary Material). It allows for the identification of the most relevant gene mutations and polymorphisms involved in arterial and deep venous thrombosis (Factor V R506Q and H1299R, Prothrombin G20210A, Methylenetetrahydrofolate Reductase (MTHFR) C677T and A1298C, Cystathionine Beta Synthase (CBS) 844ins68, Plasminogen Activated Inhibitor (PAI-1) 4G/5G, Angiotensin-Converting Enzyme (ACE) Ins/Del, Angiotensin (AGT) g.9543 T > C (M235T), Glycoprotein IIIa (GPIIIa) T1565C HPA-1 a/b, Angiotensin Receptor 1 (ATR-1) A1166C, $\beta$-fibrinogen (FGB) G455A, and Factor XIII g.7130G $>$ T (V34L) and in the cholesterol metabolism (Apolipoprotein E (ApoE)). Among the 14th SNPs included in the CVD 14 assay, five SNPs were selected based on the underlying biological plausibility discussed above: FGB 455 G/A (rs\#1,800,790), Factor V 1691 G/A (rs\#6025), Factor V 1299 H/R (\#rs770011773), Prothrombin 20,210 G/A (rs\#1,799,963), GpIIIa 1565 T/C (\#rs59189) (Table 1). DNA extraction from non-coagulated blood was performed for all samples using the DNA extraction kit, according to the manufacturer's instructions (see Supplementary Material for details). DNA target was amplified with multiplex PCR using biotinylated primers in a thermal cycler under the following conditions: $95^{\circ} \mathrm{C}$ for $2 \mathrm{~min}$, $95{ }^{\circ} \mathrm{C}$ for $30 \mathrm{~s}$, followed by denaturation at $60{ }^{\circ} \mathrm{C}$ for $30 \mathrm{~s}$, annealing at $72{ }^{\circ} \mathrm{C}$ for $45 \mathrm{~s}$, and extension at $72{ }^{\circ} \mathrm{C}$ for $5 \mathrm{~min}$ for a total of 35 cycles. The amplification products were analyzed by means of $2 \%$ agarose gel containing ethidium bromide (Sigma-Aldrich) as intercalating DNA agent. Ethidium bromide is the most used nucleic acid stain for agarose gel electrophoresis. The DNA bands were detected on a UV light box. Detection is based on the reverse-hybridization principle and was performed by using automatic instrument Profi-Blot T30/T48. Biotinylated PCR products were hybridized with allele-specific oligonucleotide probes immobilized

Table 1 Description of the selected single-nucleotide polymorphisms (SNPs)

\begin{tabular}{llllllll}
\hline Hemostatic risk allele & Approved name & Approved symbol & HGNC ID & $\begin{array}{l}\text { Chromo- } \\
\text { somal } \\
\text { location }\end{array}$ & & RS & $\begin{array}{l}\text { Point } \\
\text { mutation } \\
\text { nucleotide }\end{array}$ \\
\hline Beta-Fibrinogen 455 G/A & Fibrinogen beta chain & FGB & 3662 & $4 \mathrm{q} 31.3$ & rs1800790 & 455 & G/A \\
GpIIb/IIIa PIA2 & Integrin subunit beta 3 & ITGB3 & 6156 & $17 \mathrm{q} 21.32$ & rs5918 & 1565 & T/C \\
Factor V Leiden & Coagulation factor V & F5 & 3542 & $1 \mathrm{q} 24.2$ & rs6025 & 506 & G/A \\
Factor VH2R & Coagulation factor V & F5 & 3542 & $1 \mathrm{q} 24.2$ & rs1800595 & 1299 & T/C \\
Prothrombin G20210A & Coagulation factor II, thrombin & F2 & 3535 & $11 \mathrm{p} 11.2$ & rs1799963 & 20,210 & G/A \\
\hline
\end{tabular}

$A$, adenine; $C$, cytosine; $G$, guanine; GPIIIa, glycoprotein IIIa; HGNC, HUGO Gene Nomenclature Committee; $R S$, reference sequence; $T$, thymine 
as an array of parallel lines on membrane-based strips. The exact match between probes and amplified product generates a signal exploiting the bond between biotin and streptavidin conjugated with alkaline phosphatase and a subsequent color developer that allows for colorimetric detection.

\section{Cumulative genetic risk score}

Risk associations between each risk genotype and MS susceptibility were tested under three different genetic models: dominant, additive, and recessive models [9] (as shown in Table 3). Afterwards, for each SNP, the genotypes were coded as 0,1 , or 2 indicating the number of hemostatic risk alleles in the genotype in order to create a cumulative genetic risk score (CGRS) which included the 5 selected hemostatic risk alleles (rs\#1,800,790, rs\#6025, \#rs770011773, rs\#1,799,963, \#rs59189) (Table 1). CGRSs were calculated using the unweighted method, mirroring previous studies [10]. The unweighted CGRS of an individual is the sum of disease alleles from five SNPs obtained by adding coded genotypes (possible score range of $0-10$ ). To avoid any bias due to missing data, samples with one or more missing genotypes were not included in the genetic risk score calculations. For the unweighted genetic score analysis, genotypes from 98 cases and 60 healthy controls were considered.

\section{Statistical analysis}

For each SNP, Hardy-Weinberg equilibrium was tested in the control sample by applying chi-square test. All the demographic characteristics between MS cases and healthy controls were evaluated by applying a $t$-test for continuous data and chi-square test for discrete data. A multivariable logistic regression model was employed to assess the association between MS status and each polymorphism in the recessive and dominant model, while a multivariable multinomial logistic regression model was employed to assess the association between MS status and each polymorphism in the additive model. It is difficult to reach stringent measures of statistical significance with a small sample size, and the fairly conservative multiple testing approach, Bonferroni correction, may increase type II error (false negatives); therefore, to overcome these shortcomings, we used the unweighted CGRS. Distributions of CGRS between MS cases and controls were compared by applying nonparametric Mann-Whitney $U$ test. Moreover, $t$-test was applied to compare average CGRS between cases and controls. We employed an ordinal logistic regression model to assess the association between CGRS and MS status. Then, CGRS was divided into three groups based on the number of risk alleles in subjects (no risk: no risk alleles; low risk: one or two risk alleles; high risk: three or four risk alleles). The group with no risk alleles was used as the base outcome in the multinomial logistic regression. Testing for dose-response or testing the risk factor trend on the outcome was also applied. All the regression models were adjusted according to age and gender. Lastly, to investigate in MS patients whether the frequency of those SNPs is associated with clinical and radiological outcomes at diagnosis, a multivariable logistic model was applied. Bonferroni adjustments were performed when appropriate. Bonferroni adjustments were performed for the Mann-Whitney $U$ test applied to describe distributions of CGRS between MS cases and controls and for the $t$-test that was applied to compare average CGRS between cases and controls. Concerning the multivariate analyses, the correction for multiple comparisons was not performed for the following reasons: (i) reducing the type I error for null associations, which is the reason to correct for multiple comparisons, increases the type II error for those associations that are not null [11]; (ii) given the exploratory nature of our analyses.

Statistical analyses were performed using Stata (StataCorp. 2019. Stata Statistical Software: Release 16. College Station, TX: StataCorp LLC).

\section{Results}

\section{Association analysis of the five SNPs with MS}

Demographic and clinical characteristics of the study sample are reported in Table 2 . Controls were significantly younger than cases ( $p$ value $=0.047$ ). There was no statistically significant difference between the two groups as concerns gender. All SNP variants satisfied Hardy-Weinberg equilibrium in the control sample, with a $p$-value $>0.05$. When applying the Hardy-Weinberg equilibrium equation to FBG to the $38 \mathrm{GG}$ and to the $29 \mathrm{GA}$ carriers of the control group, an A allele prevalence of 0.22 can be estimated. This translates into an expected number of 3 AA carriers ( 1 observed). The frequencies of each variant did not show any significant difference between males and females (Table S1). In Table 3, the allelic and genotypic frequency in MS patients and healthy controls is reported. The $p$ values of the chisquare tests displayed in Table 3 for additive model refer to by 2 by 3 contingency tables and do not show the difference in risk carried by the genotype G/A compared to the genotype G/G. Therefore, our results do not suggest that the Beta-Fibrinogen $455 \mathrm{G} / \mathrm{A}$ variant is associated according to different transmission models (both additive and recessive). To highlight this, we have performed a 2 by 2 contingency table to evaluate the frequency of G/A compared to G/G between cases and controls. Results of the analysis did not show a difference between these two genotypes, as shown in Table S2a (in Supplemental Material). 
Table 2 Demographic and clinical data of MS patients and healthy controls

\begin{tabular}{llll}
\hline & $\mathrm{MS}^{\S}$ patients $(n)$ & $\begin{array}{l}\text { Healthy controls }(n) \\
68\end{array}$ & $P$ value \\
\hline $\begin{array}{l}\text { Age (years) } \\
\begin{array}{l}\text { Mean (SD) } \\
\text { Male sex }\end{array}\end{array}$ & $45.394(12.06)$ & $41.808(17.02)$ & 0.047 \\
$N$ (percentage) & $43.7 \%$ & $49.7 \%$ & 0.705 \\
$\begin{array}{l}\text { Age of onset (years) } \\
\text { Mean (SD) }\end{array}$ & $32.294(11.17)$ & - & - \\
$\begin{array}{l}\text { EDSS }{ }^{\dagger} \text { at diagnosis } \\
\text { Mean (SD) }\end{array}$ & $2.424(1.24)$ & - & - \\
$\begin{array}{l}\text { More than 9 lesions in T2 at diagnosis } \\
N \text { (percentage) }\end{array}$ & $74(64.5 \%)$ & - & - \\
$\begin{array}{l}\text { Spinal cord involvement at diagnosis } \\
N \text { (percentage) }\end{array}$ & $81(70 \%)$ & - & - \\
$\begin{array}{l}\text { Gd }+^{*} \text { lesion/s at diagnosis } \\
N \text { (percentage) }\end{array}$ & $26(25 \%)$ & - & - \\
\hline
\end{tabular}

$M S$, multiple sclerosis; EDSS, Expanded Disability Status Scale; $G D+$, gadolinium +
Concerning the FV H1299R, FV Leiden, and Prothrombin G20210A variants, no subjects in our population carried the homozygotic genotype for the risk alleles; therefore, the additive model, as well as the recessive one, is not applicable.

Results from the association analysis between individual SNPs and MS susceptibility revealed those carrying the genotype AA of the FGB polymorphism were likely to have MS as well, in the additive model (OR 8.994; 95\% CI $1.108-72.976 ; p=0.040)$ and in the recessive model (OR 10.101; 95\% CI 1.265-80.646; $p=0.029$ ), after adjustment for age and gender; however, those carrying the genotype GA did not show an increased likelihood of being in the MS status in the additive model (Table S2b). Therefore, the Beta-Fibrinogen 455 G/A polymorphism might be associated to the MS status only according to a recessive pattern of inheritance. Although more frequent in MS patients, none of the other 4 SNPs was significantly associated with MS status, after adjustment for age and sex, except for the Factor V H1299R that was significantly associated with MS status in the dominant model (OR 3.130; 95\% CI $1.012663-9.679648 ; p$ value $=0.048)$.

\section{Cumulative allele score analysis}

We examined the combined effect of these five thromboticrelated SNPs on MS susceptibility by creating a CGRS. In cases, the distribution of CGRS showed a significant shift towards higher values compared to controls (Mann-Whitney $U$ test $p$-value $<0.05$ ). However, its significance did not withstand adjustment for multiple testing using the Bonferroni correction. The average $( \pm \mathrm{SD})$ of CGRS among MS cases $(1.273 \pm 1.025 \mathrm{SD})$ was significantly higher than controls $(0.822 \pm 0.820 \mathrm{SD} ; t$-test $p$-value $=0.0029)$, comparing the two groups, and its significance withstood adjustment for Bonferroni correction $(p=0.0145)$. The distribution of CGRS between cases and controls is graphically showed in Fig. 1. As summarized in Table 4, multivariate ordinal logistic regression found a significant association between CGRS and MS status $(p=0.010)$. Then, individuals were grouped into three categories according to the weighted risk scores, no risk $(\mathrm{CGRS}=0)$, low risk $(\mathrm{CGRS}=1-2)$, and high risk (CGRS $=3-4)$. The effect sizes of the low and high groups were estimated by taking the no risk group as reference (Table 4). We observed a difference in the likelihood of being in the MS status for subjects in both categories of risk (low risk: RRR 2.193; 95\% CI 1.075-4.472; $p=0.031$; high risk RRR 8.360; 95\% CI, 1.497-107.503; $p=0.047$ ). To illustrate the effect per increase 1 risk allele, we calculated the odds ratio (OR) for trends. As the CGRS increased, the OR increased $(p=0.002)$. The ORs increased to the values of $2.126(95 \%$ CI $1.035-4.283 ; p$ value $=0.0354)$ and 12.913 (95\% CI 1.301-128.122; $p=0.0046$ ) for subjects carrying respectively 1-2 and 3-4 risk alleles (Table 4).

We did not find any significant association between the separate or combined effects of the genotypes and clinical or radiological outcomes of MS patients at diagnosis.

\section{Discussion}

In this case-control study involving subjects originating from Campania, we investigated the association between five common genetic polymorphisms involved in fibrinogenmediated hemostatic pathways with MS status. Our results revealed that FGB 455 G/A and Factor V H1299R variants might be associated with MS status, in the recessive and dominant model, respectively.

Our population of healthy subjects had a similar allele frequency of the 5 tested SNPs to that reported in healthy 


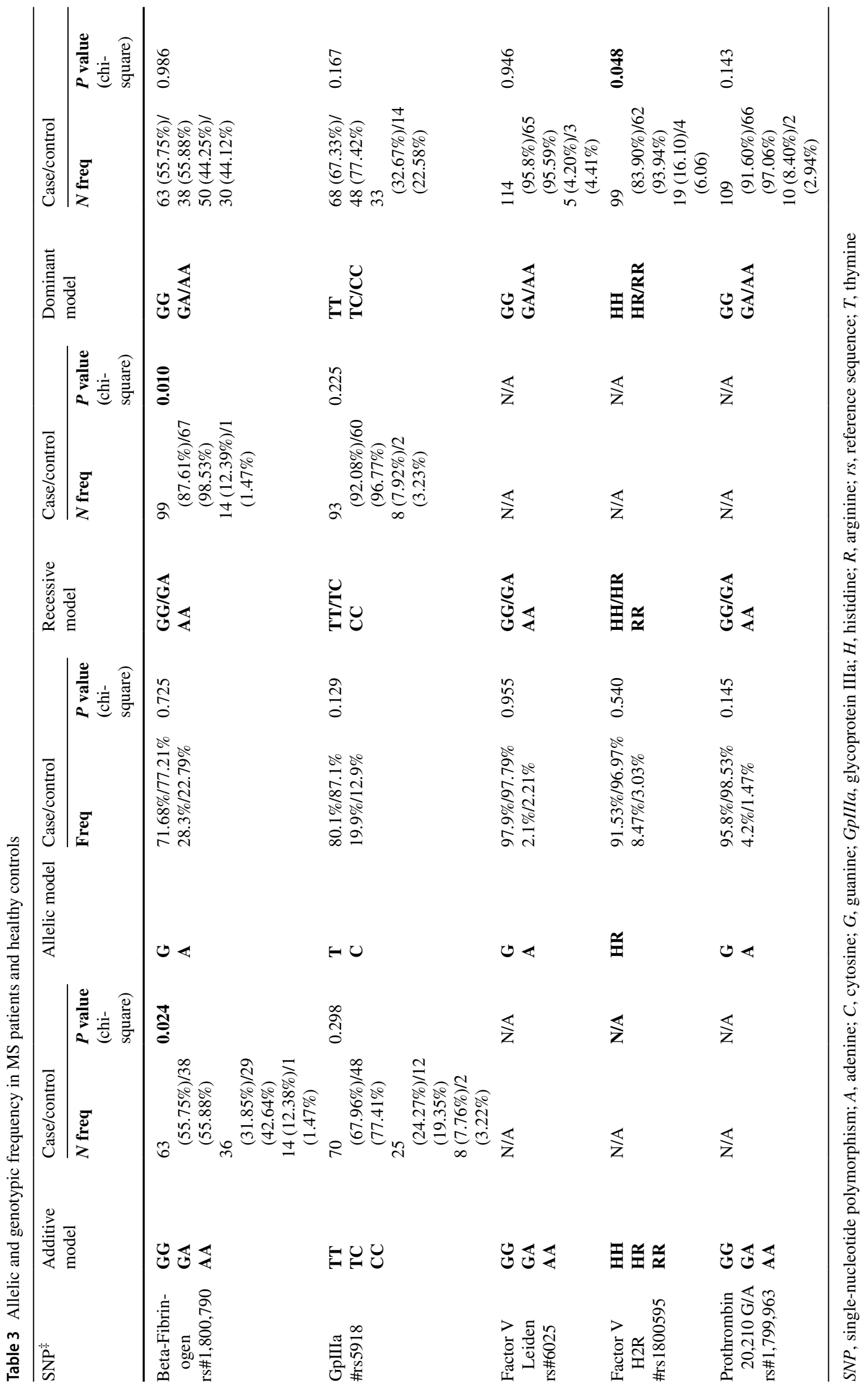


Fig. 1 Distribution of CGRS between MS patients and healthy subjects. The distribution of the cumulative genetic risk score in multiple sclerosis patients and healthy controls is represented. Allele number: number of risk alleles carried by subjects $(0,1,2,3$, or 4$)$

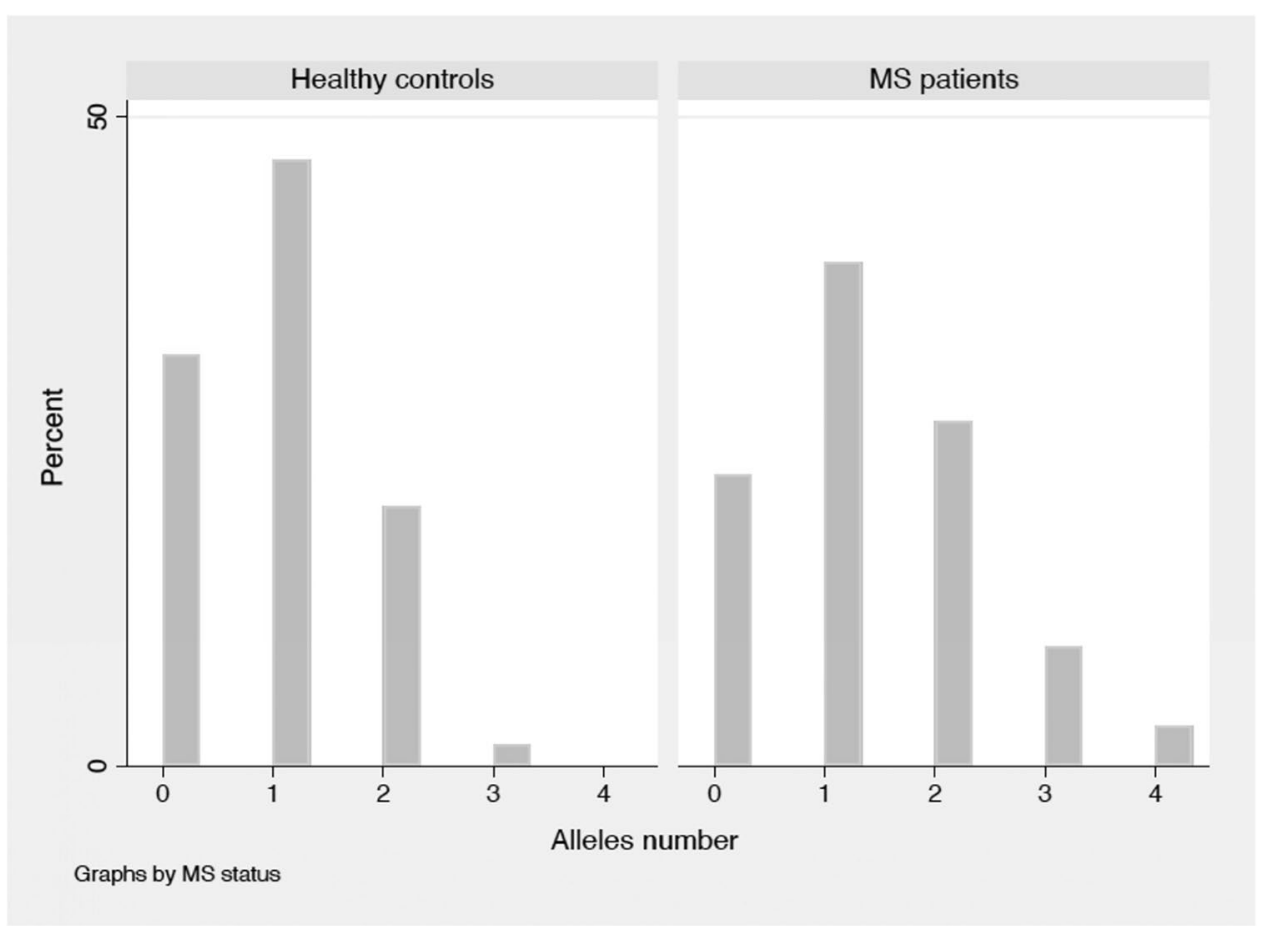

Table 4 Cumulative genetic risk score (CGRS) in MS patients compared to controls

Ordered logistic regression

\begin{tabular}{|c|c|c|c|c|c|c|}
\hline & Adjusted $O R$ & & $p$ value & & [95\% conf. interval] & \\
\hline Multiple sclerosis & 2.217 & & 0.010 & & $1.207-4.069$ & \\
\hline 1 risk allele & -0.203 & & - & & $-1.187-0.780$ & \\
\hline 2 risk alleles & 1.431 & & - & & $0.424-2.439$ & \\
\hline 3 risk alleles & 3.169 & & - & & $2.030-4.307$ & \\
\hline 4 risk alleles & 4.632 & & - & & $3.123-6.141$ & \\
\hline \multicolumn{7}{|c|}{ Multinomial logistic regression } \\
\hline Risk alleles & Adjusted RRR & & & $p$ value & [95\% conf. interval] & \\
\hline 0 & Base outcome & & & & & \\
\hline $1-2$ & 2.193 & & & $\mathbf{0 . 0 3 1}$ & $1.075-4.472$ & \\
\hline $3-4$ & 8.360 & & & 0.047 & $1.497-107.503$ & \\
\hline \multicolumn{7}{|l|}{ Test for trend } \\
\hline Risk alleles & $O R$ & $p$ value & & & & [95\% conf. interval] \\
\hline $0-1$ & 1 & - & & & & - \\
\hline 2 & 2.106 & 0.0354 & & & & $1.035-4.283$ \\
\hline $3-4$ & 12.913 & 0.0046 & & & & $1.301-128.122$ \\
\hline$P$ trend: 0.002 & & & & & & \\
\hline
\end{tabular}

$O R$, odds ratio; $R R R$, relative risk ratio

subjects from Europe, Italy (Tuscany), and Campania [12, 13], see Table S3 (Supplemental Material).

It is becoming increasingly clear that the genetic predisposition to common diseases is multifactorial, often resulting from multiple low-penetrance variants. The possible biological effect of the five markers under study in enhancing and sustaining neuroinflammation is supported by a growing body of evidence revealing the role of the fibrinogen-mediated hemostatic pathways in the pathogenesis of MS. In detail, we have studied the polymorphisms involved in the regulation of (a) fibrinogen interaction with its platelet receptor (GP IIIa P1A2); (b) fibrinogen synthesis (BetaFibrinogen $455 \mathrm{G} / \mathrm{A}$ ); and (c) fibrinogen cleavage into fibrin (Factor V Leiden, Factor V H2R, Prothrombin 20,210 G/A). 
The GP IIb/IIIa integrin is the most abundant plateletspecific glycoprotein, functioning as a receptor for ligands such as fibrinogen and von Willebrand factor, promoting platelet activation and aggregation [14]. The GPIIIa P1A variant may potentially influence both activation of the GP complexes and platelet aggregation [15, 16]. The interplay between activated platelets, endothelial cells, and infiltrating leukocyte regulates local inflammatory response and is an underlying mechanism of BBB dysfunction [17]. Several experiments have revealed an increased platelet activation, adhesiveness, and aggregation in RRMS patients [17-20]. Moreover, enhanced activation of GPIIb/IIIa, augmented formation of platelet aggregates, and increased platelet adhesiveness [21,22] were found in secondary progressive MS patients.

The other four SNPs (Factor V 1691 G/A, Factor V 1299 H/R, Prothrombin 20,210 G/A, FGB 455 G/A) influence the transcription of genes encoding for proteins involved in secondary hemostasis. Factor V Leiden and Factor V H1299R mutations may cause activated protein $\mathrm{C}$ (aPC; Factor V and Factor VIII inhibitor) resistance by reducing the susceptibility of activated Factor $\mathrm{V}$ and activated Factor VIII to aPC-mediated inactivation. The Factor II G20210A polymorphism in the 3' untranslated region of the prothrombin gene is associated with an increased level of thrombin activity [24], whereas the FGB $455 \mathrm{G} / \mathrm{A}$ polymorphism is associated with a significantly higher fibrinogen plasma level [25]. The effect on coagulation cascade of aPC-resistance, of augmented thrombin activity, and increased fibrinogen levels is the induction of a state of hypercoagulability resulting in an increased fibrin deposition [23, 24].

Significantly higher plasma levels of prothrombin have been found in MS patients compared to healthy controls [25]. Moreover, RRMS patients showed an accelerated thrombin generation compared to both PPMS and healthy controls, probably depending on the active proinflammatory state [26]. In a recent study, plasma levels of PC pathway proteins have been associated with neuroradiological measures of atrophy (volumes of total gray matter, thalamus, cortex, deep gray matter, and whole brain) [27]. Further evidence corroborating a role for thrombin and PC pathway in MS pathogenesis derives from neuropathological and experimental studies. Proteomic analysis of chronic active lesions revealed the concomitant presence of tissue factor (the initiator of coagulation cascade) and protein $\mathrm{C}$ inhibitor (PCI), suggesting that suppressed protein $\mathrm{C}$ pathway and consequently enhanced thrombin formation may play a role in the chronic neuroinflammatory process [28].

Fibrin(-ogen) was shown to be deposited in MS lesions in both RR and progressive forms. It colocalizes with microglia/macrophages and perivenous demyelination in all active lesions in RRMS, whereas it is diffusely distributed in chronically active and inactive lesions overlapping with astrocyte and axonal processes in progressive forms [29, 30].

Recently, Lee and colleagues investigated the spatial-temporal dynamics of fibrinogen deposition in marmoset EAE by creating a radiology-to-pathology linkage. They revealed fibrin deposition in the non-demyelinated inflammatory nodules, an early MS pathological finding without a magnetic resonance imaging (MRI) counterpart. Moreover, they detected fibrin(-ogen) at the border of chronic active but not in chronic inactive plaques, suggesting that fibrinogen may play a central role in sustaining chronic inflammation [5]. Moreover, fibrin(-ogen) deposition, colocalizing with axons in chronically active and inactive lesions and with neuronal loss in cortical gray matter, has revealed a linkage even with some pathological neurodegeneration findings in MS [31].

All these findings suggest the involvement of coagulation molecules, particularly fibrinogen, in the pathogenesis of MS. The complex interaction between the hemostatic and neuroinflammatory effectors seems to have a critical role in the demyelinating process since its earliest stage. An unbalanced genetic profile towards a pro-thrombotic state might constitute a predisposing condition for MS development. Indeed, the cumulative effect of the analyzed pro-thrombotic variants might influence BBB stability and, therefore, might promote coagulation molecule extravasation, deposition, and activation, which may foster the neuroinflammatory process. Due to the minimal contribution of each variant to MS susceptibility, two (FGB G455A and Factor V H1299R) of the five genetic polymorphisms under study were found to be weakly associated with MS status. In light of the synergic effects of the five SNPs in enhancing a pro-hemostatic condition, we evaluated the cumulative effects of the five genetic polymorphisms by computing a CGRS. As this is the first study to explore the association between these SNPs and MS susceptibility, CGRS was calculated using the unweighted method. Indeed, to use the weighted method, we need the true OR [10]; these estimates are not currently known. We found that MS patients carried more pro-hemostatic variants than healthy controls and that an increasing number of unfavorable alleles might increase the likelihood of being in the MS group, in the cumulative analysis; however, we should highlight that the controls were younger than cases, potentially due to an enrollment bias and this could have influenced our results. Despite this, the significance withstood the adjustment for age and sex.

We did not find correlation between the risk genotypes and the clinical-radiological outcomes of MS patients at diagnosis.

Finally, we would also highlight that none of the 5 tested SNPs (FGB 455 G/A (rs\#1,800,790) on chromosome 4; GpIIIa 1565 T/C (\#rs59189) on chromosome 17; Factor V 1691 G/A (rs\#6025) and Factor V 1299 
H/R(\#rs770011773) on chromosome 1; Prothrombin $20,210 \mathrm{G} / \mathrm{A}$ (rs\#1,799,963) on chromosome 11) is in linkage disequilibrium with some of the 200 loci known to be associated with MS [32]. Details are reported in Table S4, S5, S6, S7 and S8, in Supplemental Material.

Therefore, although the predictive values of the considered genetic variants are not backed by a highly significant statistic, our findings do not discount the involvement of these factors in MS pathogenesis and suggest evaluating these variants in a larger population-based cohort. Indeed, identifying genetic variants associated with MS could increase our knowledge of disease mechanisms; this may represent a further step towards personalized medicine by distinguishing patients according to different pathways involved in neuroinflammation.

\section{Future perspective}

This study had an explorative nature and aimed only to describe the frequency distribution of pro-thrombotic polymorphisms among patients with multiple sclerosis and healthy controls. However, since these findings would benefit from an in silico analysis, future studies are needed to assess the impact of the selected variants on the expression profile of related genes and of other genes in the same locus, using RNA expression data from brain, available in public databases.

Supplementary Information The online version contains supplementary material available at https://doi.org/10.1007/s10072-021-05608-1.

Author contribution G.A., S.B., and L.L. conceived the presented idea. G.A. developed the theory, and G.M. and A.D. wrote the manuscript. I.A. and G.D. worked out almost all of the technical details. R.P. performed statistical analysis. S.B. and L.L. encouraged G.A. to carry out the study. S.B., L.L., G.L., E.S., and M.S. supervised the findings of this work and helped with reviewing and editing of the manuscript. All authors discussed the results and contributed to the final manuscript.

Funding Open access funding provided by Universita degli Studi della Campania Luigi Vanvitelli within the CRUI-CARE Agreement.

Data availability The data that support the findings of this study are available from the corresponding author upon reasonable request.

\section{Declarations}

Ethical approval

The ethics committee of the University of Campania Luigi Vanvitelli approved this study (n. protocol 11222/20). The study conforms with the World Medical Association Declaration of Helsinki.

Consent to participate Signed informed consent to participate was obtained from all participants.

Consent for publication Signed informed consent for publication was obtained from all participants.
Competing interests The authors declare no competing interests.

Open Access This article is licensed under a Creative Commons Attribution 4.0 International License, which permits use, sharing, adaptation, distribution and reproduction in any medium or format, as long as you give appropriate credit to the original author(s) and the source, provide a link to the Creative Commons licence, and indicate if changes were made. The images or other third party material in this article are included in the article's Creative Commons licence, unless indicated otherwise in a credit line to the material. If material is not included in the article's Creative Commons licence and your intended use is not permitted by statutory regulation or exceeds the permitted use, you will need to obtain permission directly from the copyright holder. To view a copy of this licence, visit http://creativecommons.org/licenses/by/4.0/.

\section{References}

1. Reich DS, Lucchinetti CF, Calabresi PA (2018) Multiple sclerosis. N Engl J Med 378(2):169-180. https://doi.org/10.1056/NEJMr a1401483

2. D'Haeseleer M, Cambron M, Vanopdenbosch L, De Keyser J (2011) Vascular aspects of multiple sclerosis. Lancet Neurol 10:657-666. https://doi.org/10.1016/S1474-4422(11)70105-3

3. Merker M, Eichler S, Herrmann AM, Wiendl H, Kleinschnitz C, Göbel K, Meuth SG (2017) Rivaroxaban ameliorates disease course in an animal model of multiple sclerosis. J Neuroimmunol 15(313):125-128. https://doi.org/10.1016/j.jneuroim.2017.08.013

4. Plantone D, Inglese M, Salvetti M, Koudriavtseva T (2019) A perspective of coagulation dysfunction in multiple sclerosis and in experimental allergic encephalomyelitis [published correction appears in Front Neurol 2019 Mar 12;10:210]. Front Neurol 9:1175. https://doi.org/10.3389/fneur.2018.01175

5. Lee NJ, Ha SK, Sati P et al (2018) Spatiotemporal distribution of fibrinogen in marmoset and human inflammatory demyelination. Brain 141:1637-1649. https://doi.org/10.1093/brain/awy082

6. Davalos D, Ryu JK, Merlini M, Baeten KM, Le Moan N, Petersen MA, Deerinck TJ, Smirnoff DS, Bedard C, Hakozaki H, Gonias Murray S, Ling JB, Lassmann H, Degen JL, Ellisman MH, Akassoglou K (2012) Fibrinogen-induced perivascular microglial clustering is required for the development of axonal damage in neuroinflammation. Nat Commun 3:1227. https://doi.org/10.1038/ ncomms 2230

7. Ryu JK, Petersen MA, Murray SG et al (2015) Blood coagulation protein fibrinogen promotes autoimmunity and demyelination via chemokine release and antigen presentation. Nat Commun 6:8164. https://doi.org/10.1038/ncomms9164

8. Ryu JK, Rafalski VA, Meyer-Franke A et al (2018) Fibrin-targeting immunotherapy protects against neuroinflammation and neurodegeneration. Nat Immunol 19:1212-1223

9. Zhao F, Song M, Wang Y, Wang W (2016) Genetic model. J Cell Mol Med 20(4):765. https://doi.org/10.1111/jcmm.12751

10. Liyanarachchi S, Wojcicka A, Li W et al (2013) Cumulative risk impact of five genetic variants associated with papillary thyroid carcinoma. Thyroid 23(12):1532-1540. https://doi.org/10.1089/ thy. 2013.0102

11. Rothman KJ (1990) No adjustments are needed for multiple comparisons. Epidemiology 1(1):43-46

12. Alexander TA, Machiela MJ (2020) LDpop: an interactive online tool to calculate and visualize geographic LD patterns. BMC Bioinformatics 21(1):14. https://doi.org/10.1186/s12859-020-3340-1

13. Cernera G, Comegna M, Gelzo M et al (2021) Molecular analysis of prothrombotic gene variants in patients with acute ischemic stroke and with transient ischemic attack. Medicina 57(7):723 
14. Kucharska-Newton AM, Monda KL, Campbell S et al (2011) Association of the platelet GPIIb/IIIa polymorphism with atherosclerotic plaque morphology: the Atherosclerosis Risk in Communities (ARIC) study. Atherosclerosis 216(1):151-156. https:// doi.org/10.1016/j.atherosclerosis.2011.01.038

15. Di Castelnuovo A, de Gaetano G, Donati MB, Iacoviello L (2001) Platelet glycoprotein receptor IIIa polymorphism PLA1/PLA2 and coronary risk: a meta-analysis. Thromb Haemost 85(4):626-633

16. Todinova S, Komsa-Penkova R, Krumova S, Taneva SG, Golemanov G, Georgieva G, Tonchev P, Tsankov B, Beshev L, Balashev K, Andreeva TD (2017) PlA2 Polymorphism in glycoprotein IIb/IIIa modulates the morphology and nanomechanics of platelets. Clin Appl Thromb Hemost 23(8):951-960. https://doi.org/ 10.1177/1076029616687847

17. Saluk-Bijak J, Dziedzic A, Bijak M (2019) Pro-thrombotic activity of blood platelets in multiple sclerosis. Cells 1;8(2):110. https:// doi.org/10.3390/cells8020110

18. Marcos-Ramiro B, Oliva Nacarino P, Serrano-Pertierra E et al (2014) Microparticles in multiple sclerosis and clinically isolated syndrome: effect on endothelial barrier function. BMC Neurosci 15:110. https://doi.org/10.1186/1471-2202-15-110

19. Sheremata WA, Jy W, Horstman LL, Ahn YS, Alexander JS, Minagar A (2008) Evidence of platelet activation in multiple sclerosis. J Neuroinflammation 5:27. https://doi.org/10.1186/ 1742-2094-5-27

20. Lock C, Hermans G, Pedotti R et al (2002) Gene-microarray analysis of multiple sclerosis lesions yields new targets validated in autoimmune encephalomyelitis. Nat Med 8:500-508. https:// doi.org/10.1038/nm0502-500

21. Morel A, Bijak M, Miller E, Rywaniak J, Miller S, Saluk J (2015) Relationship between the increased haemostatic properties of blood platelets and oxidative stress level in multiple sclerosis patients with the secondary progressive stage. Oxid Med Cell Longev 2015:240918. https://doi.org/10.1155/2015/240918

22. Morel A, Rywaniak J, Bijak M, Miller E, Niwald M, Saluk J (2017) Flow cytometric analysis reveals the high levels of platelet activation parameters in circulation of multiple sclerosis patients. Mol Cell Biochem 430:69-80. https://doi.org/10.1007/ s11010-017-2955-7

23. Tiedje V, Dunkler D, Ay C, Horvath B et al (2011) The role of fibrinogen plasma levels, the $-455 \mathrm{G}>\mathrm{A}$ fibrinogen and the factor XIII A subunit (FXIII-A) Val34Leu polymorphism in cancer-associated venous thrombosis. Thromb Haemost 106(5):908-913. https://doi.org/10.1160/TH11-04-0278

24. Demirci FY, Dressen AS, Kammerer CM, Barmada MM, Kao AH, Ramsey-Goldman R, Manzi S, Kamboh MI (2011) Functional polymorphisms of the coagulation factor II gene (F2) and susceptibility to systemic lupus erythematosus. J Rheumatol 38(4):652-657. https://doi.org/10.3899/jrheum.100728

25. Gobel K, Kraft P, Pankratz S et al (2016) Prothrombin and factor $\mathrm{X}$ are elevated in multiple sclerosis patients. Ann Neurol 80:946951. https://doi.org/10.1002/ana.24807

26. Parsons ME, O'Connell K, Allen S et al (2017) Thrombin generation correlates with disease duration in multiple sclerosis (MS): novel insights into the MS-associated prothrombotic state. Mult Scler J Exp Transl Clin 3:2055217317747624. https://doi.org/10. $1177 / 2055217317747624$

27. Magliozzi R, Hametner S, Facchiano F et al (2019) Iron homeostasis, complement, and coagulation cascade as CSF signature of cortical lesions in early multiple sclerosis. Ann Clin Transl Neurol 6:2150-2163. https://doi.org/10.1002/acn3.50893

28. Han MH, Hwang SI, Roy DB et al (2008) Proteomic analysis of active multiple sclerosis lesions reveals therapeutic targets. Nature 451:1076-1081. https://doi.org/10.1038/nature06559

29. Vos CM, Geurts JJ, Montagne L et al (2005) Blood-brain barrier alterations in both focal and diffuse abnormalities on postmortem MRI in multiple sclerosis. Neurobiol Dis 20:953-960. https://doi. org/10.1016/j.nbd.2005.06.012

30. Marik C, Felts PA, Bauer J, Lassmann H, Smith KJ (2007) Lesion genesis in a subset of patients with multiple sclerosis: a role for innate immunity? Brain 130:2800-2815. https://doi.org/10.1093/ brain/awm236

31. Petersen MA, Ryu JK, Akassoglou K (2018) Fibrinogen in neurological diseases: mechanisms, imaging and therapeutics. Nat Rev Neurosci 19:283-301. https://doi.org/10.1038/nrn.2018.13

32. International Multiple Sclerosis Genetics Consortium (2019) Multiple sclerosis genomic map implicates peripheral immune cells and microglia in susceptibility. Science 365(6460):eaav7188

Publisher's note Springer Nature remains neutral with regard to jurisdictional claims in published maps and institutional affiliations. 